\title{
Comparison of Gadoteric Acid and Gadobutrol for Detection as Well as Morphologic and Dynamic Characterization of Lesions on Breast Dynamic Contrast-Enhanced Magnetic Resonance Imaging
}

\author{
Diane M. Renz, MD, * Tahir Durmus, MD, * Joachim Böttcher, MD, + Matthias Taupitz, MD, * \\ Felix Diekmann, MD, $\neq$ Alexander Huppertz, MD, \& Alexander Pfeil, MD,// Martin H. Maurer, MD,* \\ Florian Streitparth, MD, * Ulrich Bick, MD, * Bernd Hamm, MD, * and Eva M. Fallenberg, MD*
}

\begin{abstract}
Objective: In contrast to conventional breast imaging techniques, one major diagnostic benefit of breast magnetic resonance imaging (MRI) is the simultaneous acquisition of morphologic and dynamic enhancement characteristics, which are based on angiogenesis and therefore provide insights into tumor pathophysiology. The aim of this investigation was to intraindividually compare 2 macrocyclic MRI contrast agents, with low risk for nephrogenic systemic fibrosis, in the morphologic and dynamic characterization of histologically verified mass breast lesions, analyzed by blinded human evaluation and a fully automatic computer-assisted diagnosis (CAD) technique.

Materials and Methods: Institutional review board approval and patient informed consent were obtained. In this prospective, single-center study, 45 women with 51 histopathologically verified (41 malignant, 10 benign) mass lesions underwent 2 identical examinations at $1.5 \mathrm{~T}$ (mean time interval, 2.1 days) with $0.1-\mathrm{mmol} \mathrm{kg}^{-1}$ doses of gadoteric acid and gadobutrol. All magnetic resonance images were visually evaluated by 2 experienced, blinded breast radiologists in consensus and by an automatic CAD system, whereas the morphologic and dynamic characterization as well as the final human classification of lesions were performed based on the categories of the Breast imaging reporting and data system MRI atlas. Lesions were also classified by defining their probability of malignancy (morpho-dynamic index; $0 \%-100 \%$ ) by the CAD system. Imaging results were correlated with histopathology as gold standard.
\end{abstract}

Results: The CAD system coded 49 of 51 lesions with gadoteric acid and gadobutrol (detection rate, 96.1\%); initial signal increase was significantly higher for gadobutrol than for gadoteric acid for all and the malignant coded lesions $(P<0.05)$. Gadoteric acid resulted in more postinitial washout curves and fewer continuous increases of all and the malignant lesions compared with gadobutrol (CAD hot spot regions, $P<0.05$ ). Morphologically, the margins of the malignancies were different between the 2 agents, whereas gadobutrol demonstrated more spiculated and fewer smooth margins $(P<0.05)$. Lesion classifications by the human observers and by the morpho-dynamic

Received for publication August 28, 2013; and accepted for publication, after revision, December 24, 2013.

From the *Department of Radiology, Charite University Medicine Berlin, Berlin; $\dagger$ Institute of Diagnostic and Interventional Radiology, SRH Clinic Gera, Gera; Institute of Diagnostic Radiology, St. Joseph Stift, Bremen; §Imaging Science Institute, Berlin; and ||Department of Internal Medicine III, Friedrich-SchillerUniversity, Jena University Hospital, Jena, Germany.

Conflicts of interest and source of funding: The study was initiated by the investigators and was supported by a research grant from Bayer Healthcare, Leverkusen, Germany. A part of the study was supported by a research grant from Guerbet, Roissy CdG, Cedex, France. However, the data and the results of the study were independently from any grants obtained, and the investigators had exclusive control of all data. Thus, there was no conflict of interest regarding the study.

Drs Renz and Durmus contributed equally in this study.

Reprints: Diane M. Renz, MD, Department of Radiology, Charité University Medicine Berlin, Charitéplatz 1, 10117 Berlin, Germany. E-mail: diane.renz@charite.de.

Copyright (C) 2014 by Lippincott Williams \& Wilkins

ISSN: 0020-9996/14/4907-0474 index compared with the histopathologic results did not significantly differ between gadoteric acid and gadobutrol.

Conclusions: Macrocyclic contrast media can be reliably used for breast dynamic contrast-enhanced MRI. However, gadoteric acid and gadobutrol differed in some dynamic and morphologic characterization of histologically verified breast lesions in an intraindividual, comparison. Besides the standardization of technical parameters and imaging evaluation of breast MRI, the standardization of the applied contrast medium seems to be important to receive best comparable MRI interpretation.

Key Words: breast MRI, contrast medium, gadoteric acid, gadobutrol, breast cancer, CAD

\section{(Invest Radiol 2014;49: 474-484)}

B reast carcinoma is the most common cancer in women worldwide. ${ }^{1}$ Breast dynamic contrast-enhanced magnetic resonance imaging (DCE-MRI) is gaining wide acceptance as an accurate imaging technique for detection, staging, and monitoring of invasive breast carcinomas. ${ }^{2-8}$ Compared with conventional breast imaging modalities, one major benefit of DCE-MRI is the possibility to acquire morphologic and dynamic enhancement characteristics, which are based on angiogenesis and provide insights into tumor pathophysiology. ${ }^{4,9,10}$ Thus, the intravenous application of contrast media is essential for reliable lesion detection and diagnosis by breast DCEMRI. ${ }^{4,9,10}$ To standardize imaging evaluation, Breast imaging reporting and data system (BI-RADS) MRI atlas, which considers morphologic and dynamic parameters, has been established and reported to reveal acceptable interobserver agreement. ${ }^{11-14}$

To further minimize interobserver variability, computer-assisted diagnosis (CAD) systems for breast DCE-MRI are increasingly integrated into clinical practice for the assessment of kinetic enhancement features. ${ }^{15}$ There is high clinical interest in incorporating also morphologic assessment into CAD systems and in linking computerextracted features to the descriptors of the BI-RADS MRI atlas. Some of the CAD systems for breast DCE-MRI combine morphologic and dynamic parameters, showing promising results, whereas different MRI contrast media have been used. ${ }^{16-20}$ However, intraindividual, visually evaluated comparison studies found differences in signal enhancement, detection, and differentiation between malignant and benign breast lesions according to the MRI contrast agent used. ${ }^{21-24}$

Extracellular gadolinium (Gd)-based MRI contrast media are categorized into macrocyclic and linear agents owing to their chemical structures. $^{25,26}$ The macrocyclic molecules form a rigid ring system, in which Gd ions are caged, whereas the linear chelates wrap around the Gd ions. ${ }^{25,26}$ Compared with linear agents, macrocyclic compounds release less free Gd ions; this higher stability becomes relevant with regard to nephrogenic systemic fibrosis in patients with impaired renal function, as the macrocyclic chelates are associated with lower risk of developing this severe systemic disorder. ${ }^{26-30}$ Gadoteric acid (Gd-DOTA; Dotarem; Guerbet, Roissy CdG, Cedex, 
France) and gadobutrol (Gd-DO3A-butriol; Gadovist; Bayer Healthcare, Leverkusen, Germany) are both macrocyclic contrast media with currently lower worldwide application for breast DCE-MRI compared with conventional linear agents, such as gadopentetate dimeglumine (Gd-DTPA; Magnevist; Bayer Healthcare). The purpose of this study was to prospectively and intraindividually compare $0.1 \mathrm{mmol} \mathrm{kg}^{-1}$ body weight doses of gadoteric acid and gadobutrol for the detection and characterization of histologically confirmed malignant and benign breast masses; to provide as much as possible objective interpretation, magnetic resonance (MR) images were evaluated by blinded human visual evaluation as well as by a unique fully automatic CAD system, which analyzed dynamic and morphologic parameters and linked these features to the BI-RADS descriptors.

\section{MATERIALS AND METHODS}

\section{Study Standards and Design}

This prospective investigation (clinical trial phase IIIb) was performed in accordance with the Declaration of Helsinki and was approved by the local ethics committee and the Federal Institute for Drugs and Medical Devices (Bonn, Germany). Patients were consecutively enrolled over a period of 8 months; all patients gave written informed consent before study participation.

Female patients were included if they were at least 18 years of age and had suspicious findings on x-ray mammography and/or ultrasonography (BI-RADS categories 4 and 5). The patients were not part of the government-funded mammography screening program but received $\mathrm{x}$-ray mammography and ultrasonography for clinical indications, such as palpable mass, or pain in the breast. After conventional breast imaging, the patients were scheduled for DCE-MRI for further diagnostic imaging and treatment planning. Another important inclusion criterion was the presence of mass breast lesions, as the used CAD system has been solely evaluated in detail for mass lesions and the morphologic description of lesions by the CAD system corresponded to the BI-RADS categories of masses. ${ }^{18}$ The reference standard was the histopathologic confirmation of the mass lesions, whereas experienced breast pathologists performed histopathologic diagnosis. The lesions were categorized by using the World Health Organization classification of malignant and benign breast tumors; the grading of carcinomas was specified as well (grade, G1), moderately (G2), and poorly (G3) differentiated cancers.

Exclusion criteria were the following:

-Contraindications to MRI, for example, pacemaker, claustrophobia, severe obesity;

-Application of any contrast medium within 24 hours before receiving gadoteric acid or gadobutrol and between the 2 MRI examinations;

-History of anaphylactoid or anaphylactic reaction to any contrast medium;

-Pregnant or lactating women;

-Clinically unstable condition and/or requirement of emergency treatment and/or physical or mental status, which interfered with the signing of the informed consent; and

-Chronic kidney disease stage 3 or greater, that means glomerular filtration rate of lower than $60 \mathrm{~mL} \mathrm{~min}^{-1}$, as 2 contrast media administrations have to be applied.

All patients received one DCE-MRI examination with gadoteric acid and one with gadobutrol; the technical MRI parameters of the 2 examinations were identical. Patients were randomized prospectively into 2 groups (A or B) before breast DCE-MRI. In group A, gadoteric acid was administered for the first and gadobutrol for the second examination; group B received the agents in reverse order. The interval between the 2 MRI examinations was between 24 hours and 7 days; within this period, the patients did not undergo systemic or local treatment including interventional procedure, such as breast biopsy, surgery, radiation and/or chemotherapy.

\section{MRI Protocol}

Breast DCE-MRI examinations were performed with predefined standardized parameters on a 1.5-T system (Magnetom Avanto; Siemens Healthcare, Erlangen, Germany) using a 4-channel bilateral breast coil with the patient in prone position. Threedimensional (3D) gradient-echo T1-weighted sequences were acquired precontrast and postcontrast by using the following parameters: repetition time, 7.5 milliseconds; echo time, 4.8 milliseconds; flip angle, $25^{\circ}$; field of view (FOV), 320 to $360 \mathrm{~mm}$; acquisition matrix, $384 \times 384$; and spatial resolution, $0.7 \times 0.7 \times 2 \mathrm{~mm}^{3}$. Each sequence comprised of 72 slices in transverse orientation. Acquisition time for one T1-weighted sequence was 59 to 77 seconds depending on breast size. Twenty seconds after beginning of contrast medium injection, dynamic scanning was continued with the same sequence parameters and under identical tuning conditions; 5 postcontrast series were acquired with identical time intervals. Unenhanced images were subtracted from postcontrast images. In addition, transverse T2-weighted turbo-spin-echo sequences were performed before contrast medium administration (repetition time, 6750 milliseconds; echo time, 80 milliseconds; flip angle, $150^{\circ}$; FOV, 320-360 mm; acquisition matrix, $448 \times 448$; spatial resolution, $0.7 \times 0.7 \times 2.5 \mathrm{~mm}^{3}$ ).

\section{Contrast Medium Administration}

Gadoteric acid is hydrophilic and negatively charged, with a Gd concentration of $0.5 \mathrm{~mol} \mathrm{~L}^{-1}$. The hydrophilic, neutral contrast medium gadobutrol contains twice the $\mathrm{Gd}$ concentration $\left(1 \mathrm{~mol} \mathrm{~L}^{-1}\right)$. Both agents possess high thermodynamic, kinetic, and conditional stability as well as low osmolality and viscosity. ${ }^{25,26}$ Gadobutrol reveals an approximately 1.5 -fold higher $\mathrm{T} 1$ relaxivity $\left(5.2 \mathrm{~L} \mathrm{mmol}^{-1} \mathrm{~s}^{-1}\right.$ at $37^{\circ} \mathrm{C}$ in plasma at $\left.1.5 \mathrm{~T}\right)$ compared with $3.6 \mathrm{~L} \mathrm{mmol}^{-1} \mathrm{~s}^{-1}$ of gadoteric acid. ${ }^{31}$ Both contrast media have been proven as well tolerated with low rates of adverse events. ${ }^{32,33}$

Both agents were automatically administered in $0.1-\mathrm{mmol} \mathrm{kg}^{-1}$ doses by a power injector with a flow rate of $2 \mathrm{~mL} \mathrm{~s}^{-1}$. The contrast medium injection was followed by a $0.9 \%$ sodium chloride solution with the same flow rate. Because of its doubled Gd concentration and the equimolar dose, the administered volume of gadobutrol was half that of gadoteric acid. The volume of the saline flush was therefore adapted [volume saline flush, $30 \mathrm{~mL}$ for gadoteric acid; volume saline flush for gadobutrol, $30 \mathrm{~mL}+$ (volume gadoteric acid - volume gadobutrol) $\mathrm{mL}$ ] to equalize the total amount of injection volumes and, thus, the injection times in both MRI examinations.

\section{Analysis of MR Images}

All MR images were visually evaluated by 2 experienced breast radiologists ( 7 and 10 years of experience in breast MRI) in consensus and by an automatic CAD system (Breast MRI Carebox; Bracco Imaging, Milan, Italy). For visual imaging evaluation, the 2 human blinded readers analyzed the MR images considering the following viewpoints: detection of contrast-enhanced breast lesions, detailed morphologic characterization of lesions, visual dynamic interpretation, and final lesions' classification. The MR imaging analysis of the human readers as well as of the CAD system was performed based on the guidelines of the BI-RADS MRI atlas. ${ }^{11}$ The human observers categorized the lesions as benign or malignant following the ordinal scale of the BI-RADS classification: 1, no pathologic enhancement; 2 , definitely benign; 3 probably benign; 4 , suspicious abnormality; and 5, highly suggestive of malignancy. All MRI examinations were evaluated by the readers in a blinded, anonymized manner to clinical and radiological data and in a randomized order, without knowledge of which contrast media had been applied. Both human observers reviewed the data set for the first time for study purpose, as they were not involved in the clinical imaging diagnosis of the patients, to prevent recall bias. The patients 
TABLE 1. Histologic Characteristics of Mass Breast Lesions

No. Lesions

\begin{tabular}{lr}
\hline Malignant lesions ( $\mathrm{n}=41)$ & 32 \\
Invasive ductal carcinoma & 4 \\
$\quad$ Well differentiated (G1) & 19 \\
Moderately differentiated (G2) & 9 \\
$\quad$ Poorly differentiated (G3) & 5 \\
Invasive lobular carcinoma & 1 \\
$\quad$ Well differentiated (G1) & 4 \\
$\quad$ Moderately differentiated (G2) & 1 \\
Invasive papillary carcinoma (moderately differentiated, G2) & 1 \\
Invasive mucinous carcinoma (moderately differentiated, G2) & 2 \\
Ductal carcinoma in situ & 1 \\
$\quad$ Intermediate grade (G2) & 1 \\
$\quad$ High grade (G3) & \\
Benign lesions (n = 10) & 6 \\
Fibroadenoma & 6 \\
Adenosis & 2 \\
Atypical ductal hyperplasia & 1 \\
Papilloma & 1 \\
\hline
\end{tabular}

who fulfilled the specific inclusion criteria were preselected and enrolled by a clinician radiologist dedicated to breast imaging, who analyzed the x-ray mammographic, ultrasonographic, and MR images during clinical routine.

In addition to the description and classification of contrastenhancing lesions, the 2 blinded human observers assessed background enhancement (BE) independently for all MRI examinations during the study imaging evaluation. This BE, owing to hormonal effects and mastopathic proliferative disease, was visually determined by using the ordinal scale of Baltzer et $\mathrm{al}^{34}$ : grade 0 , no relevant $\mathrm{BE}$; grade 1 , moderate $\mathrm{BE}$; and grade 2 , distinct $\mathrm{BE}$.

For the CAD imaging evaluation, the dynamic series were transferred as anonymized DICOM (Digital Imaging and Communications in Medicine) files to the automatic CAD system. The specific characteristic of the used CAD technique was that it automatically detected contrast-enhancing breast lesions and performed dynamic as well as morphologic characterization of the lesions and linked these descriptions to features, laid down in the BI-RADS MRI atlas. ${ }^{11,18}$ The CAD system has been recently described and evaluated in detail, showing that it can reliably distinguish between benign and malignant breast mass lesions with a diagnostic accuracy of $93.5 \%{ }^{18}$ Possible motion artifacts were reduced by a 3D registration algorithm; contrast-enhancing lesions were detected by a segmentation technique with an adaptive threshold. ${ }^{18}$ For morphologic characterization of detected lesions, the CAD system extracted 13 parameters: volume, maximum and minimum diameter, surface area, surface-to-volume ratio, compactness, characteristic of margin, homogeneity, average signal intensity, deviation of signal intensities, presence of spiculations, adjacent vessels, or central necrosis. ${ }^{18}$ These parameters were used to provide morphologic descriptors of masses according to the BI-RADS MRI lexicon and were included in the final lesions' classification of the CAD system. ${ }^{18}$

The signal intensity time curves, achieved by CAD analysis, were divided in an initial phase during the first 2 postcontrast sequences (graduated in 50\% intervals of relative signal increase) and a postinitial phase (continuous increase, plateau phenomenon, and washout curve). ${ }^{9,11,18}$ The CAD system performed the dynamic analysis by calculating signal intensity time curves of the whole lesions, considering average signal intensity values, and the hot spot regions, focusing on the location within the lesions $(3 \times 3$ voxels $)$ with the highest signal enhancement during the first 2 postcontrast sequences. Signal intensity time curves were displayed for the whole lesions and the hot spot regions. For the final lesions' classification, the following 8 dynamic parameters were additionally calculated for the entire lesions and the hot spot regions: relative enhancements (1-5) between precontrast and each of the 5 postcontrast sequences, maximum peak value of the signal intensity time curve, relative signal increase within the first 2 postcontrast sequences, and presence of washout sign. All extracted morphologic and dynamic parameters were integrated for final lesions' classification by the CAD system; this calculated morpho-dynamic index (MDI) assessed the probability of detected lesions to be malignant, ranging from $0 \%$ to $100 \%$. $^{18}$

\section{Statistical Evaluation}

The statistical analysis, including sample size calculation for valid contrast media comparison, was scheduled and approved by a statistician experienced in this field. The evaluations of the MRI examinations using gadoteric acid versus gadobutrol were compared with each other and correlated with the gold standard of histopathologic findings. Statistical analysis was performed by using SPSS version 17.0 for Windows (SPSS, Chicago, IL). The prevalence of all morphologic and dynamic parameters was determined for both MRI examinations. Student $t$ tests were used to examine significant differences between means of 2 samples with normal distribution. For analyzing further possible differences, Wilcoxon rank sum tests were calculated for ordinal data, and Pearson $\chi^{2}$ tests were performed for nominal data. For correlation analyses, Pearson (interval parameters), Spearman rank (ordinal parameters), and Cramer $V$ coefficients (nominal data) were used. Receiver operating characteristic analysis was performed to calculate the diagnostic accuracy (area under the receiver operating characteristic curve) of the breast MRI examinations. $P$ values $<0.05$ (2-sided) were considered in all statistical tests to indicate significances.

\section{RESULTS}

\section{Patients and Histopathology}

Forty-seven women with histopathologically verified mass lesions were enrolled in the study; in the intraindividual contrast media comparison, solely the histopathologically confirmed mass lesions were considered. If the patients presented additional lesions that were not histopathologically proven after the MRI examinations, these lesions were not included in the comparison study. Two of 47 patients had to be excluded, as they refused the second MRI examination. Thus, 45 women fulfilled the inclusion criteria $(\mathrm{n}=23$ group $\mathrm{A}, \mathrm{n}=22$ group $\mathrm{B}$ ). The mean (SD) time interval between the 2 MRI examinations was $2.1(1.2)$ days; the exact intervals were as follows: 1 day, $\mathrm{n}=13$ patients; 2 days, $\mathrm{n}=22 ; 3$ days, $\mathrm{n}=6 ; 4$ days, $\mathrm{n}=2 ; 5$ days, $\mathrm{n}=1$, and 7 days, $\mathrm{n}=1$. The 45 patients had a mean (SD) age of 49.9 (12.1) years (range, 30-70 years); 23 women $(51.1 \%)$ were premenopausal and $22(48.9 \%)$ were postmenopausal.

The $\mathrm{x}$-ray mammographic and ultrasonographic findings were categorized as BI-RADS 4 in 27 of 45 patients $(60.0 \%)$ and as 5 in 18 of 45 patients $(40.0 \%)$. As all women had BI-RADS categorization 4 or 5 on conventional imaging modalities, they could not discontinue possible hormonal medication for a longer time interval before DCE-MRI to avoid retarding possible cancer treatment initiation. Thus, 12 women received hormonal medication directly before the MRI examinations ( 8 patients contraceptives and 4 hormone replacement treatment). In 37 patients, malignant lesions were identified, which presented as masses; histopathology found invasive breast cancer in 35 of 37 women and ductal carcinoma in situ in 2 of 37 patients. Four of 37 patients revealed bifocal, unilateral, invasive carcinomas. Thus, 41 mass lesions were histopathologically confirmed as 
malignant in 37 patients. Histologic types and tumor grading of these 41 malignant lesions are summarized in Table 1. Regarding breast cancer therapy, more than half of 37 patients $(n=20 ; 54.1 \%)$ underwent breast conservation followed by radiation. Ten women $(27.0 \%)$ received mastectomy, and $18.9 \%(\mathrm{n}=7)$ received neoadjuvant chemotherapy with subsequent breast surgery.

Eight of 45 patients presented benign mass lesions, which were histologically confirmed after the second DCE-MRI examination. Two patients had histopathologically confirmed malignant and benign mass lesions. One mass lesion (atypical ductal hyperplasia) was surgically removed, as the 58 -year-old patient underwent mastectomy because of invasive ductal, G2 carcinoma in the same breast. A 39-year-old woman received breast conservation because of invasive ductal, G3 carcinoma in the left breast and synchronous surgical extirpation of a fibroadenoma in the contralateral breast. Thus, 10 benign mass lesions could be included with different histopathologic entities, as shown in Table 1.

\section{Lesion Detection and Size}

Thirty-nine of 41 malignant and all 10 benign mass lesions were detected by the CAD method on the MRI examinations with gadoteric acid and also with gadobutrol, corresponding to a detection rate each of $96.1 \%$ (49 detected of 51 malignant and benign lesions). Two malignancies were missed by the CAD technique on both MRI examinations (identical lesions by gadoteric acid and gadobutrol). Both lesions could visually be observed by the human readers with gadoteric acid and gadobutrol. One missed lesion (7 mm mean size on MRI) was an invasive ductal cancer lesion (G3) in a 63-year-old patient (contrast medium group B) with dense breast, experiencing bifocal carcinoma, whereas her second, 12-mm-sized cancer lesion was detected by CAD on both examinations. The missed lesion presented an initial signal increase of $50 \%$ to $100 \%$ followed by continuous enhancement, assessed by manual region of interest analysis, on both MRI examinations, similar to the surrounding dense glandular breast tissue. The second missed lesion by CAD was an invasive ductal cancer (G3; $13 \mathrm{~mm}$ mean size on MRI) in a 37-year-old patient (group A) who received hormonal contraceptives and revealed severe BE (grade 2) by using gadoteric acid and gadobutrol. The lesion demonstrated an initial signal increase of $100 \%$ to $150 \%$ followed by plateau phenomenon, measured by manual region of interest analysis, on both MRI examinations. The automated lesion detection was hampered because of the severe $\mathrm{BE}$ in this patient.

The remaining 44 patients in whom histopathologically verified lesions could be detected by the CAD system revealed similar $\mathrm{BE}$ for gadoteric acid and gadobutrol. On both MRI examinations, 10 of 44 women $(22.7 \%$ ) presented moderate BE (grade 1 ) and 7 of 44 patients $(15.9 \%)$ demonstrated distinct BE (grade 2). However, $\mathrm{BE}$ was not strong enough to impair the automated lesion detection in these patients.

Assessment of lesion diameters by the CAD method and by the observers resulted in slightly lower sizes of the 49 detected mass lesions for gadoteric acid compared with gadobutrol (Table 2). These size differences between the 2 MRI examinations were not significant, neither for CAD nor for visual analysis (Student $t$ tests; $P>0.05$ ). For lesions that were surgically removed subsequently to the MRI examinations $(\mathrm{n}=35)$, mean diameters were compared with histologically determined sizes. The MRI examinations with gadoteric acid resulted in lower, not significantly different, assessments compared with the histologically verified sizes; by using gadobutrol, the assessments were slightly, not significantly, higher (Table 2). As shown in Figure 1, MRI size assessments by using gadoteric acid and gadobutrol are both in good accordance with histopathologically verified diameters of breast lesions.

\section{Dynamic Lesion Characterization}

As the CAD system provided a very differentiated dynamic analysis, performing separate evaluation for whole lesion and hot spot region, Tables 3 to 5 demonstrate the $\mathrm{CAD}$ evaluations in detail. The initial signal increase of all, malignant, and benign lesions differed between the 2 distinct MRI examinations, with lower values using gadoteric acid than gadobutrol for the entire lesions (Fig. 2) and the hot spot regions. These differences reached significance for the entire lesions and hot spot regions of all masses $(n=49$; Table 3$)$ and the malignancies $(n=39$; Table 4$)$, but not for the benign lesions solely $(n=10$; Table 5). Focusing on postinitial enhancement courses, MRI examinations with gadoteric acid revealed more washout curves and fewer continuous increases compared with gadobutrol (Tables 3-5). These differences were significant for the hot spot regions of all lesions and the malignant masses (Tables 3 and 4).

TABLE 2. Assessed Sizes of Mass Breast Lesions

\begin{tabular}{|c|c|c|c|c|}
\hline & Mean, mm & Median, mm & $\mathrm{SD}, \mathbf{m m}$ & Interquartile Range, mm \\
\hline \multicolumn{5}{|l|}{ All lesions $(n=49)$} \\
\hline \multicolumn{5}{|l|}{ CAD evaluation } \\
\hline MRI examinations with gadoteric acid & 25.0 & 21.0 & 13.0 & 21.0 \\
\hline MRI examinations with gadobutrol & 26.6 & 23.0 & 13.7 & 18.5 \\
\hline \multicolumn{5}{|l|}{ Visual evaluation } \\
\hline MRI examinations with gadoteric acid & 25.3 & 21.0 & 13.5 & 19.0 \\
\hline Histologically verified size & 26.4 & 22.0 & 14.3 & 20.0 \\
\hline \multicolumn{5}{|l|}{ CAD evaluation } \\
\hline MRI examinations with gadoteric acid & 25.4 & 21.0 & 13.3 & 21.0 \\
\hline MRI examinations with gadobutrol & 27.0 & 22.0 & 13.2 & 16.0 \\
\hline \multicolumn{5}{|l|}{ Visual evaluation } \\
\hline MRI examinations with gadoteric acid & 25.7 & 22.0 & 13.3 & 17.0 \\
\hline
\end{tabular}

CAD indicates computer-assisted diagnosis; MRI, magnetic resonance imaging. 


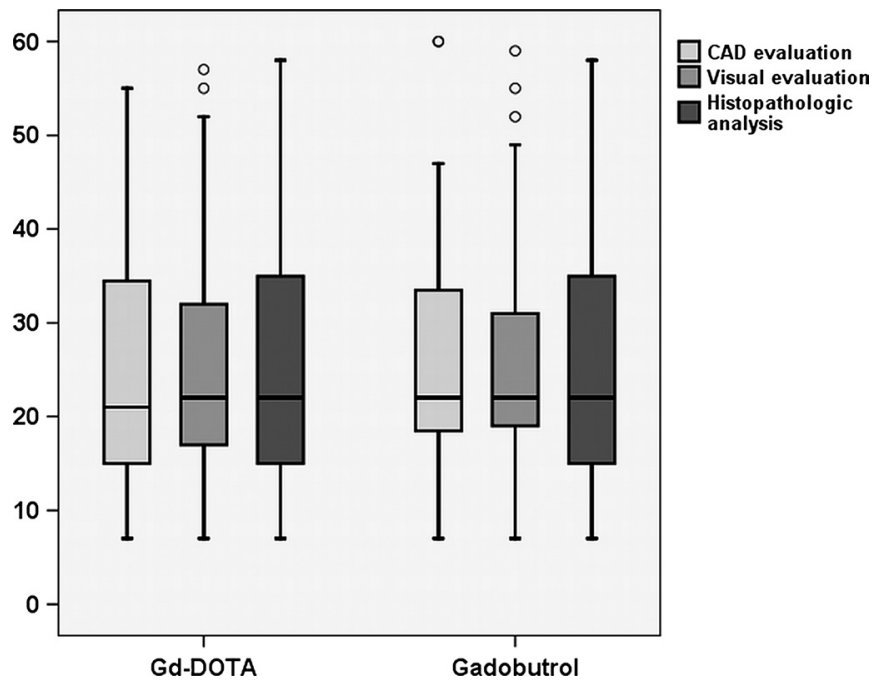

FIGURE 1. Box plots present the size assessments of lesions, which were surgically removed subsequently to the MRI examinations $(n=35)$. The lesion diameters have been determined for both MRI examinations (with gadoteric acid and gadobutrol) automatically by the CAD system and visually by the human readers and compared with the histopathologically verified sizes.

\section{Morphologic Lesion Characterization}

The morphologic description by the CAD system was highly consistent with the assessment of the human readers. Regarding the shape of the 49 malignant and benign masses, Spearman rank correlation coefficient was 0.839 for gadoteric acid $(P<0.001)$ and 0.823 for gadobutrol $(P<0.001)$. The margins of the 49 lesions, assessed by the readers, also showed high consistency with the CAD evaluation: Spearman rank correlation coefficient $\mathrm{rs}=0.856$ $(P<0.001)$ for gadoteric acid and rs $=0.863(P<0.001)$ for gadobutrol. The correlation coefficient between human and CAD assessment of the internal enhancement of the 49 lesions was Cramer $V=0.781(P<0.001)$ for gadoteric acid and Cramer $V=0.798(P<0.001)$ for gadobutrol.

As the morphologic description of breast lesions is commonly performed in clinical routine by visual, not by CAD, evaluation, the findings of the 2 human observers are presented in detail. The morphologic parameters of the 10 histologically verified benign lesions were determined identically by the human readers on both MRI examinations (Fig. 3). Their shape was assessed as follows: $n=7$ round/oval and $\mathrm{n}=3$ lobulated. Six benign lesions presented smooth and 4 showed irregular margins. The internal enhancement of the benign lesions was determined as homogeneous $(n=5)$, heterogeneous $(\mathrm{n}=3)$, and dark internal septations $(\mathrm{n}=2)$.

In contrast to the benign lesions, some of the 39 malignant masses showed different morphologic descriptions on the examinations with gadoteric acid versus gadobutrol, as analyzed by the CAD system and the human observers. Regarding shape, 4 of 39 malignancies $(10.3 \%)$ were classified differently by the human readers: 2 lesions were determined as round/oval by using gadoteric acid and as irregular with gadobutrol, and 2 lesions were defined as lobulated with gadoteric acid and as irregular with gadobutrol. The differences in the shape assessment were not significant (Table 6). However, the margins of the malignancies were determined as significantly different between the 2 MRI examinations by the observers (Table 6); descriptions of margins were discrepant for 9 of 39 (23.1\%) malignant lesions by using gadoteric acid versus gadobutrol: smooth versus spiculated, $\mathrm{n}=6$; smooth versus irregular, $\mathrm{n}=1$ (Fig. 2); and
TABLE 3. Signal Intensity Time Courses of 49 Histologically Verified Mass Lesions ( $n=39$ Malignant, $n=10$ Benign) as Calculated by the CAD System

\begin{tabular}{|c|c|c|c|}
\hline $\begin{array}{l}\text { Dynamic Signal } \\
\text { Intensity Time Courses }\end{array}$ & $\begin{array}{c}\text { MRI } \\
\text { Examinations } \\
\text { With } \\
\text { Gadoteric Acid }\end{array}$ & $\begin{array}{c}\text { MRI } \\
\text { Examinations } \\
\text { With } \\
\text { Gadobutrol }\end{array}$ & $P$ (2-Sided) \\
\hline \multicolumn{4}{|l|}{ Entire lesions } \\
\hline Initial phase & & & $<0.05$ \\
\hline $50 \%-100 \%$ & $7(14.3)$ & $2(4.1)$ & \\
\hline $100 \%-150 \%$ & $11(22.4)$ & $11(22.4)$ & \\
\hline $150 \%-200 \%$ & $13(26.5)$ & $9(18.4)$ & \\
\hline $200 \%-250 \%$ & $10(20.4)$ & $12(24.5)$ & \\
\hline $250 \%-300 \%$ & $6(12.2)$ & $8(16.3)$ & \\
\hline $300 \%-400 \%$ & $2(4.1)$ & $3(6.1)$ & \\
\hline $400 \%-500 \%$ & $0(0.0)$ & $2(4.1)$ & \\
\hline$>500 \%$ & $0(0.0)$ & $2(4.1)$ & \\
\hline Postinitial phase & & & NS \\
\hline Continuous increase & $13(26.5)$ & $18(36.7)$ & \\
\hline Plateau phenomenon & $17(34.7)$ & $15(30.6)$ & \\
\hline Washout curve & $19(38.8)$ & $16(32.7)$ & \\
\hline \multicolumn{4}{|l|}{ Hot spot regions } \\
\hline Initial phase & & & $<0.05$ \\
\hline $50 \%-100 \%$ & $1(2.0)$ & $0(0.0)$ & \\
\hline $100 \%-150 \%$ & $3(6.1)$ & $2(4.1)$ & \\
\hline $150 \%-200 \%$ & $8(16.3)$ & $5(10.2)$ & \\
\hline $200 \%-250 \%$ & $12(24.5)$ & $9(18.4)$ & \\
\hline $250 \%-300 \%$ & $13(26.5)$ & $8(16.3)$ & \\
\hline $300 \%-400 \%$ & $9(18.4)$ & $15(30.6)$ & \\
\hline $400 \%-500 \%$ & $1(2.0)$ & $5(10.2)$ & \\
\hline$>500 \%$ & $2(4.1)$ & $5(10.2)$ & \\
\hline Postinitial phase & & & $<0.05$ \\
\hline Continuous increase & $7(14.3)$ & $14(28.6)$ & \\
\hline Plateau phenomenon & $13(26.5)$ & $15(30.6)$ & \\
\hline Washout curve & $29(59.2)$ & $20(40.8)$ & \\
\hline
\end{tabular}

Numbers in parentheses are percentages; the percentages may not add up to $100.0 \%$ because of rounding. The differences were evaluated by using Wilcoxon rank sum tests.

CAD indicates computer-assisted diagnosis; MRI, magnetic resonance imaging; NS, nonsignificant.

irregular versus spiculated, $\mathrm{n}=2$. Regarding the parameter internal enhancement pattern, 6 malignancies $(15.4 \%)$ were determined differently $(P>0.05$; Table 6$)$; their assessment was as follows for gadoteric acid versus gadobutrol: rim enhancement versus heterogeneous, $\mathrm{n}=3$; heterogeneous versus rim enhancement, $\mathrm{n}=2$; and dark internal septations versus enhancing internal septations, $\mathrm{n}=1$.

\section{Classification of Lesions}

The diagnostic accuracy of gadoteric acid and gadobutrol was similar between the 2 MRI examinations for the human lesions' classification based on the BI-RADS categorization. Compared with gadoteric acid, the MDI calculations of the CAD system were slightly higher by using gadobutrol for all lesions, the malignancies, and the benign lesions (Table 7, Fig. 4). However, the mean differences between the MDI calculations of gadoteric acid versus gadobutrol were not significant (all lesions, $P=0.710$; malignancies, $P=0.661$; and benign lesions, $P=0.871$ ). Distinguishing malignant from benign lesions by the MDI, the diagnostic accuracy was also similar for gadoteric acid (93.2\%; 95\% confidence interval, 
TABLE 4. Signal Intensity Time Courses of 39 Malignant Mass Lesions as Calculated by the CAD System

\begin{tabular}{|c|c|c|c|}
\hline $\begin{array}{l}\text { Dynamic Signal } \\
\text { Intensity Time Courses }\end{array}$ & $\begin{array}{c}\text { MRI } \\
\text { Examinations } \\
\text { With } \\
\text { Gadoteric Acid }\end{array}$ & $\begin{array}{c}\text { MRI } \\
\text { Examinations } \\
\text { With } \\
\text { Gadobutrol }\end{array}$ & $\begin{array}{c}P \\
\text { (2-Sided) }\end{array}$ \\
\hline \multicolumn{4}{|l|}{ Entire lesions } \\
\hline Initial phase & & & $<0.05$ \\
\hline $50 \%-100 \%$ & $4(10.3)$ & $1(2.6)$ & \\
\hline $100 \%-150 \%$ & $7(17.9)$ & $5(12.8)$ & \\
\hline $150 \%-200 \%$ & $12(30.8)$ & $7(17.9)$ & \\
\hline $200 \%-250 \%$ & $9(23.1)$ & $12(30.8)$ & \\
\hline $250 \%-300 \%$ & $5(12.8)$ & $8(20.5)$ & \\
\hline $300 \%-400 \%$ & $2(5.1)$ & $2(5.1)$ & \\
\hline $400 \%-500 \%$ & $0(0.0)$ & $2(5.1)$ & \\
\hline$>500 \%$ & $0(0.0)$ & $2(5.1)$ & \\
\hline Postinitial phase & & & NS \\
\hline Continuous increase & $6(15.4)$ & $9(23.1)$ & \\
\hline Plateau phenomenon & $14(35.9)$ & $14(35.9)$ & \\
\hline Washout curve & $19(48.7)$ & $16(41.0)$ & \\
\hline \multicolumn{4}{|l|}{ Hot spot regions } \\
\hline Initial phase & & & $<0.01$ \\
\hline $100 \%-150 \%$ & $2(5.1)$ & $1(2.6)$ & \\
\hline $150 \%-200 \%$ & $4(10.3)$ & $2(5.1)$ & \\
\hline $200 \%-250 \%$ & $10(25.6)$ & $5(12.8)$ & \\
\hline $250 \%-300 \%$ & $12(30.8)$ & $8(20.5)$ & \\
\hline $300 \%-400 \%$ & $8(20.5)$ & $14(35.9)$ & \\
\hline $400 \%-500 \%$ & $1(2.6)$ & $4(10.3)$ & \\
\hline$>500 \%$ & $2(5.1)$ & $5(12.8)$ & \\
\hline Postinitial phase & & & $<0.05$ \\
\hline Continuous increase & $2(5.1)$ & $7(17.9)$ & \\
\hline Plateau phenomenon & $10(25.6)$ & $13(33.3)$ & \\
\hline Washout curve & $27(69.2)$ & $19(48.7)$ & \\
\hline
\end{tabular}

Numbers in parentheses are percentages; the percentages may not add up to $100.0 \%$ because of rounding. The differences were evaluated by using Wilcoxon rank sum tests.

$\mathrm{CAD}$ indicates computer-assisted diagnosis; MRI, magnetic resonance imaging; NS, nonsignificant.

$86.1 \%-100.0 \%)$ and gadobutrol $(93.1 \%$; 95\% confidence interval, $85.9 \%-100.0 \%$ ).

\section{DISCUSSION}

To optimize the diagnostic yield of breast DCE-MRI, the focus of investigations has mainly set on imaging parameters and technical advances. ${ }^{4,12,35-40}$ Considerably fewer publications have compared different contrast media for breast MRI using intraindividual, prospective, blinded study designs. ${ }^{21-24}$ Differences in dynamic signal enhancement, correct detection, and classification of malignant and benign breast lesions have been reported between gadobenate dimeglumine (Gd-BOPTA) and Gd-DTPA. ${ }^{21-23}$ The higher signal intensities for gadobenate dimeglumine are supposed to rely mainly on its approximately 2 -fold higher $\mathrm{T} 1$ relaxivity compared with gadopentetate dimeglumine..$^{2-23,41,42}$ In our clinical trial, gadobutrol reached higher initial signal intensities, statistically significant for all and also exclusively the malignant lesions. One explanation might be the approximately 1.5 -fold higher $\mathrm{T} 1$ relaxivity of gadobutrol compared with gadoteric acid. The doubled Gd concentration per unit volume is another possible reason; the $1.0 \mathrm{M}$ chelate gadobutrol has been shown to allow a narrower bolus profile, resulting in an increased, more compact intravascular concentration and, consecutively a sharper bolus peak during first pass compared with $0.5 \mathrm{M}$ agents. ${ }^{32,43,44}$ In our investigation, the effect of the higher gadobutrol concentration was presumably diminished by the administration of a larger amount of sodium chloride solution.

High contrast medium concentration exists early in the extravascular extracellular space (EES) of tumors, as smallmolecular MRI contrast media with weights less than $1000 \mathrm{Da}$, such as gadoteric acid and gadobutrol, rapidly diffuse from intravascular to EES, especially through malignant microvessels with endothelial leakages. ${ }^{25,45}$ In general, the principal pathway and mechanism of breast tumor enhancement are assumed to be similar for all small-molecular contrast media. ${ }^{10,25,45}$ The higher signal enhancement of gadobutrol, both during first pass and lasting up to several minutes, resulting in more continuous increases and fewer washout curves, is in concordance with published results. Anzalone et $\mathrm{al}^{46}$ found significantly higher signal enhancement and contrast-to-noise ratio of cerebral neoplasms for gadobutrol than gadoteric acid several minutes after contrast medium administration in an intraindividual comparison. Attenberger et $\mathrm{al}^{47}$ analyzed signal enhancement in a rat brain glioma model; signal-to-noise ratio and contrast-to-noise ratio in the tumor were higher for gadobutrol compared with gadoteric acid and gadopentetate dimeglumine at 5 time points ( 1 minute postcontrast and 4 consecutive 2 -minutes' intervals thereafter).

TABLE 5. Signal Intensity Time Courses of 10 Benign Mass Lesions as Calculated by the CAD System

\begin{tabular}{|c|c|c|c|}
\hline $\begin{array}{l}\text { Dynamic Signal } \\
\text { Intensity Time Courses }\end{array}$ & $\begin{array}{c}\text { MRI } \\
\text { Examinations } \\
\text { With } \\
\text { Gadoteric Acid }\end{array}$ & $\begin{array}{c}\text { MRI } \\
\text { Examinations } \\
\text { With } \\
\text { Gadobutrol }\end{array}$ & $\begin{array}{c}P \\
\text { (2-Sided) }\end{array}$ \\
\hline \multicolumn{4}{|l|}{ Entire lesions } \\
\hline Initial phase & & & NS \\
\hline $50 \%-100 \%$ & $3(30.0)$ & $1(10.0)$ & \\
\hline $100 \%-150 \%$ & $4(40.0)$ & $6(60.0)$ & \\
\hline $150 \%-200 \%$ & $1(10.0)$ & $2(20.0)$ & \\
\hline $200 \%-250 \%$ & $1(10.0)$ & $0(0.0)$ & \\
\hline $250 \%-300 \%$ & $1(10.0)$ & $0(0.0)$ & \\
\hline $300 \%-400 \%$ & $0(0.0)$ & $1(10.0)$ & \\
\hline Postinitial phase & & & NS \\
\hline Continuous increase & $7(70.0)$ & $9(90.0)$ & \\
\hline Plateau phenomenon & $3(30.0)$ & $1(10.0)$ & \\
\hline \multicolumn{4}{|l|}{ Hot spot regions } \\
\hline Initial phase & & & NS \\
\hline $50 \%-100 \%$ & $1(10.0)$ & $0(0.0)$ & \\
\hline $100 \%-150 \%$ & $1(10.0)$ & $1(10.0)$ & \\
\hline $150 \%-200 \%$ & $4(40.0)$ & $3(30.0)$ & \\
\hline $200 \%-250 \%$ & $2(20.0)$ & $4(40.0)$ & \\
\hline $250 \%-300 \%$ & $1(10.0)$ & $0(0.0)$ & \\
\hline $300 \%-400 \%$ & $1(10.0)$ & $1(10.0)$ & \\
\hline $400 \%-500 \%$ & $0(0.0)$ & $1(10.0)$ & \\
\hline Postinitial phase & & & NS \\
\hline Continuous increase & $5(50.0)$ & $7(70.0)$ & \\
\hline Plateau phenomenon & $3(30.0)$ & $2(20.0)$ & \\
\hline Washout curve & $2(20.0)$ & $1(10.0)$ & \\
\hline
\end{tabular}

Numbers in parentheses are percentages. The differences were evaluated by using Wilcoxon rank sum tests.

CAD indicates computer-assisted diagnosis; MRI, magnetic resonance imaging; NS, nonsignificant. 

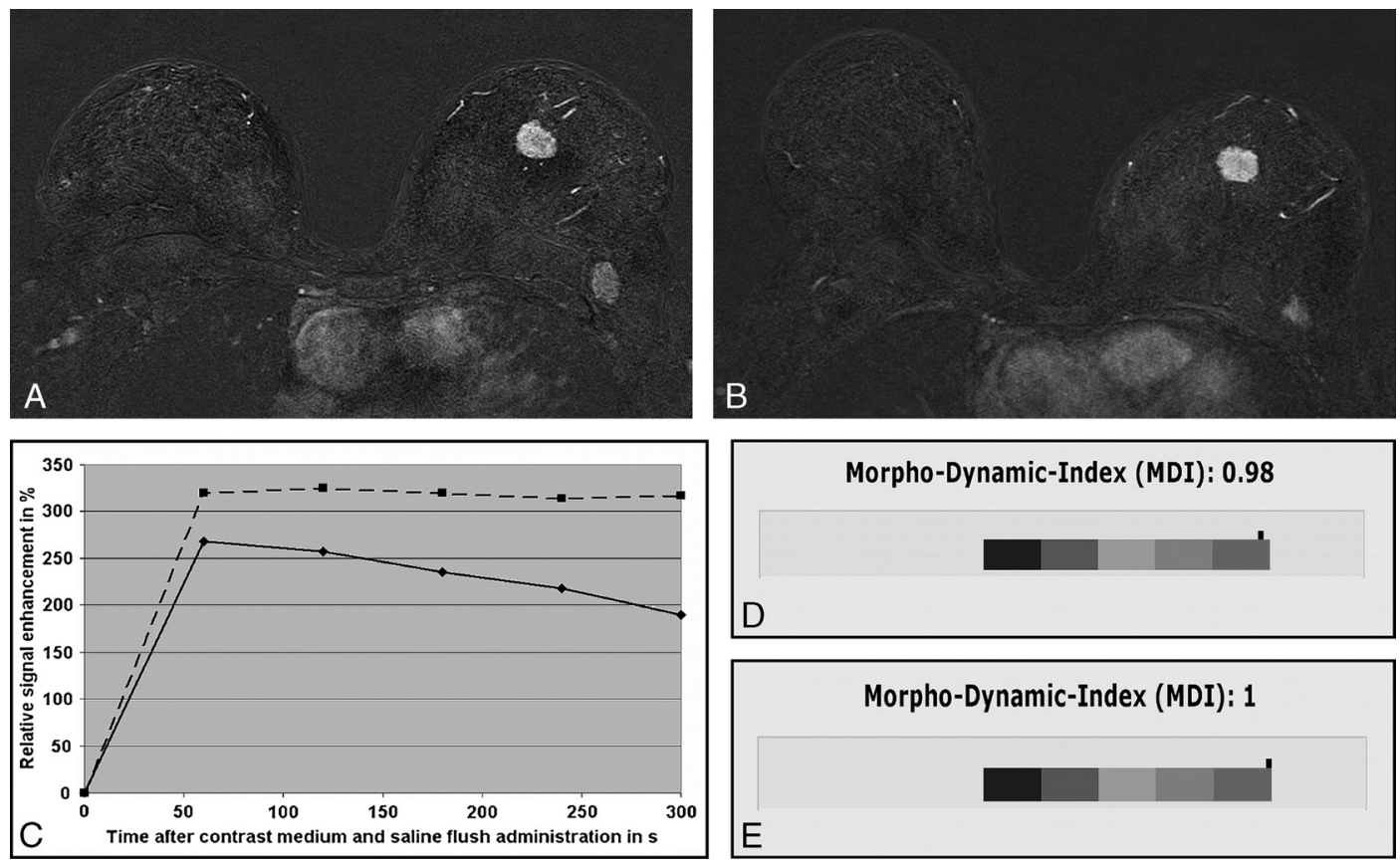

FIGURE 2. MRI examinations with gadoteric acid (A) and gadobutrol (B) of a 58-year-old patient with moderately differentiated, invasive ductal carcinoma in the left breast. The first postcontrast subtraction images show lower initial signal increase of the entire lesion for gadoteric acid (A) compared with gadobutrol (B). The morphologic characterization of the carcinoma has been determined by the human observers in both examinations with an oval shape and a heterogeneous signal enhancement. The margin has been assessed by the observers as smooth with gadoteric acid (A) and as irregular by using gadobutrol (B). In addition, both MRI examinations demonstrate a lymph node, suspicious for malignant infiltration, in the left axillary. The CAD system displayed signal intensity time curve of the whole lesion (C), demonstrating the relative signal intensity in percentage over time in seconds, with an initial enhancement of greater than $250 \%$ followed by washout curve (signal decrease, $>10 \%$ ) in the MRI examination with gadoteric acid (continuous curve); the signal intensity time curve of the whole lesion was displayed as initial enhancement of greater than $300 \%$, followed by plateau phenomenon (signal intensity, $\pm 10 \%$ ) in the MRI examination with gadobutrol (discontinuous curve). The probability of malignancy (MDI) was assessed by the CAD system as $98 \%$ in the MRI examination with gadoteric acid (D) and as $100 \%$ by using gadobutrol (E).

The persistent higher signal intensities after gadobutrol administration might be caused partly by differences in T1 relaxivities; however, relaxivity is not the only property of a contrast agent that influences imaging parameters. The higher rate of continuous increases accompanied by fewer washout curves can pharmacokinetically be explained that gadobutrol accumulates to a higher extent in the EES of breast carcinomas than gadoteric acid. As the molecular weight of gadobutrol $(605 \mathrm{Da})$ is only slightly higher compared with that of gadoteric acid (559 Da), this property might be of minor importance for EES accumulation. ${ }^{25,33,44}$ Unlike gadobenate dimeglumine, gadoteric acid and gadobutrol are generally considered as non-proteinbinding. ${ }^{25,32,33,48}$ However, Vogler et $\mathrm{al}^{48}$ identified an amount of $2.7 \%$ protein binding in gadobutrol, although calculations of low protein binding are prone to substantial measurement errors. ${ }^{48}$ If, in fact, gadobutrol possesses some weak protein binding in contrast to gadoteric acid and thus interacts with proteins of the extracellular tumoral matrix, this mechanism could also contribute to the postinitial signal intensity differences.

The major cause for the postinitial differences, significant for all and the malignant lesions, however, might be the negative charge of gadoteric acid versus the neutrality of gadobutrol. The extracellular matrix of malignant and some benign tumors possess glycosaminoglycan macromolecules with negatively ionized side groups. ${ }^{49}$ Imaging studies of articular cartilage, whose extracellular matrix is rich in glycosaminoglycans, revealed that the concentration of glycosaminoglycans is negatively correlated with signal enhancement, if negatively charged contrast media are used. ${ }^{50,51}$ In contrast, neutral agents slowly diffuse into cartilage tissue independently of glycosaminoglycan concentration. ${ }^{51}$ The negative charge of gadoteric acid might therefore be particularly responsible for lower accumulation in the tumoral EES. This hypothesis is in concordance with the findings of a recently published intraindividual comparison of gadobutrol versus gadobenate dimeglumine; Pediconi et $\mathrm{al}^{24}$ found more continuous signal intensity time curves of malignant breast lesions by using gadobutrol compared with the ionic gadobenate dimeglumine, evaluated by 2 readers: $28.6 \%$ versus $15.7 \%$ (reader 1 ) and $35.2 \%$ versus $28.4 \%$ (reader 2 ). However, these differences did not reach statistical significance in this multicenter investigation. ${ }^{24}$

The higher signal enhancement and the increased accumulation in the tumoral EES of gadobutrol might be responsible for the slightly larger lesion diameters (with very good accordance to histopathologically verified sizes) and the higher number of breast carcinomas with spiculated margins combined with fewer smooth boundaries. As a consequence, the malignant infiltration of surrounding tissues, resulting in ill-defined boundaries, should be clearly visualized with gadobutrol. This finding is in accordance with Pediconi et al, ${ }^{24}$ who reported that significantly more malignancies were assessed as non-massenhancements (boundaries more ill-defined compared with masses) for gadobutrol than for gadobenate dimeglumine. The final lesion classification by the CAD system (MDI) did not differ between gadobutrol and gadoteric acid, as the higher initial signal increase and the morphologic shape description of malignancies were assessed in a 

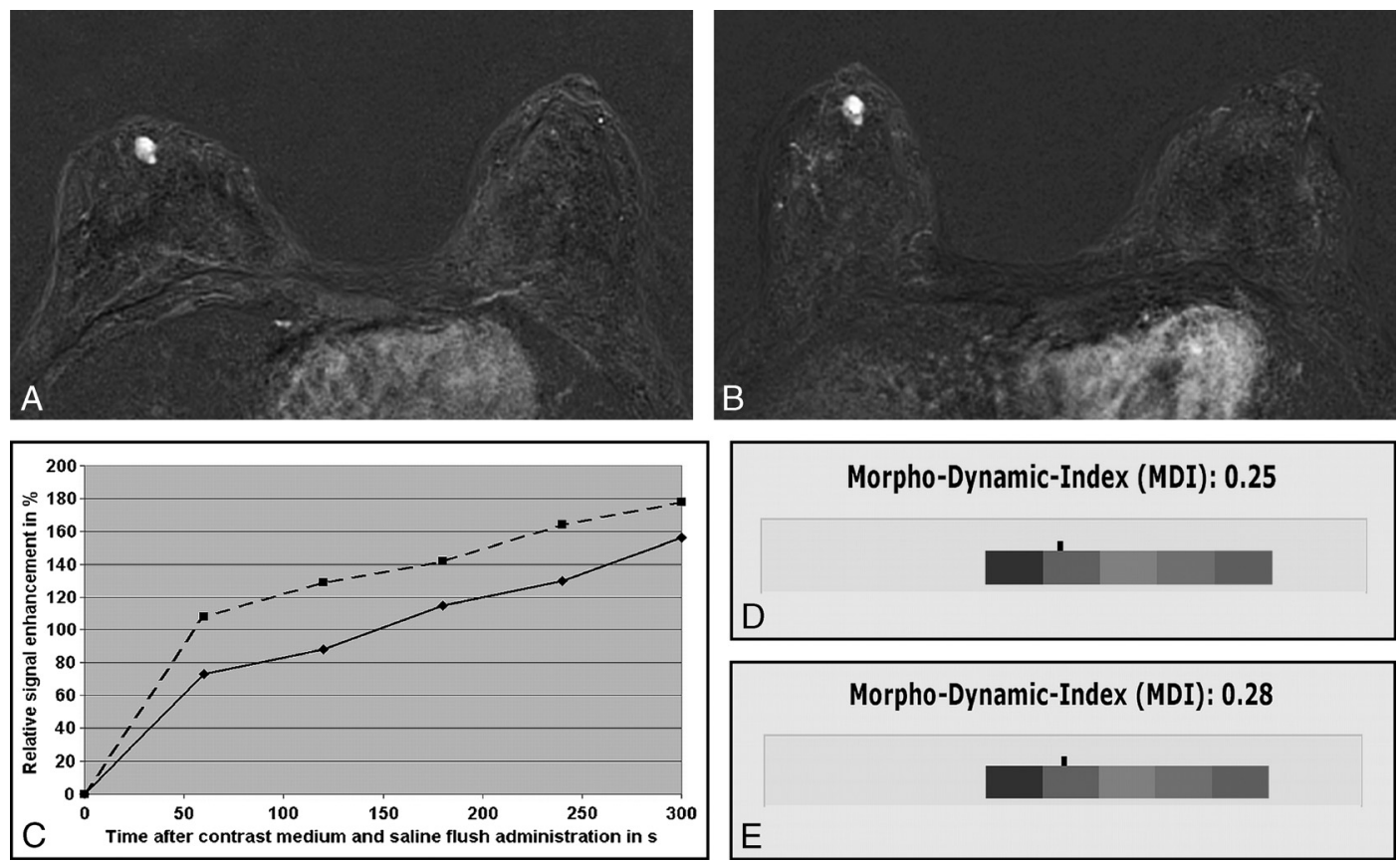

FIGURE 3. MRI examinations with gadoteric acid (A) and gadobutrol (B) of a 39-year-old patient with histologically verified fibroadenoma in the right breast. In the first postcontrast subtraction images (A, gadoteric acid; $B$, gadobutrol), the morphologic characterization of the fibroadenoma has been determined by the human observers in both examinations with an oval shape, a smooth margin, and a heterogeneous signal enhancement. The CAD system displayed the signal intensity time curve of the whole lesion (C), demonstrating the relative signal intensity in percentage over time in seconds, with an initial enhancement between $50 \%$ and $100 \%$, followed by continuous increase (signal increase, $>10 \%$ ) in the MRI examination with gadoteric acid (continuous curve); the signal intensity time curve of the whole lesion was displayed as initial enhancement between $100 \%$ and $150 \%$, followed by continuous increase in the MRI examination with gadobutrol (discontinuous curve). The probability of malignancy (MDI) was assessed by the CAD system as $25 \%$ in the MRI examination with gadoteric acid (D) and as $28 \%$ by using gadobutrol (E).

higher rate as typically malignant by using gadobutrol; however, gadoteric acid resulted in higher quantity of characteristic washout curves of malignant lesions.
Despite the standardized interpretation of breast MR images by using the internationally accepted BI-RADS atlas, the MRI technical protocol does vary considerably between institutions

TABLE 6. Morphologic Parameters of 39 Malignant Mass Lesions as Determined by the Human Observers

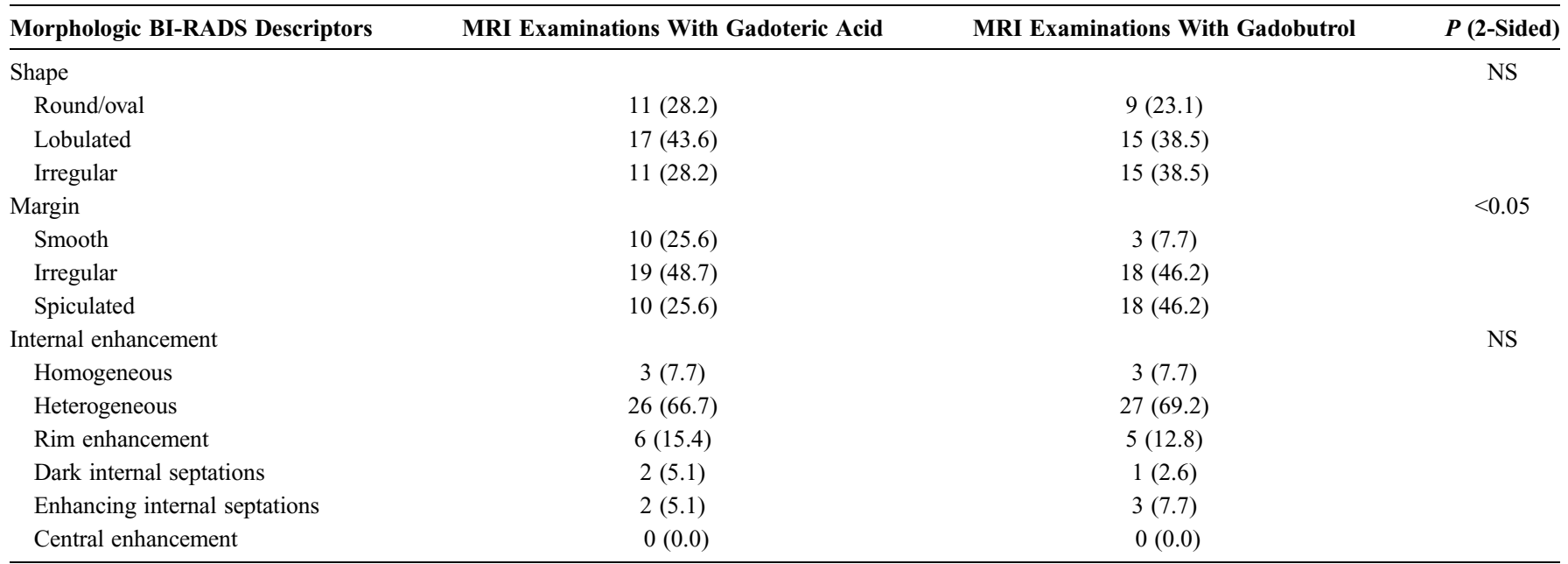

Numbers in parentheses are percentages; the percentages may not add up to $100.0 \%$ because of rounding. The differences were evaluated by using Wilcoxon rank sum tests (shape and margin) and Pearson $\chi^{2}$ test (internal enhancement).

BI-RADS indicates Breast imaging reporting and data system; MRI, magnetic resonance imaging; NS, nonsignificant. 
TABLE 7. Morpho-Dynamic Index of Mass Lesions as Calculated by the CAD System

\begin{tabular}{|c|c|c|c|c|}
\hline & Mean, \% & Median, \% & SD, \% & Interquartile Range, \% \\
\hline \multicolumn{5}{|l|}{ All lesions $(n=49)$} \\
\hline MRI examinations with gadoteric acid & 73.9 & 82.0 & 24.5 & 34.0 \\
\hline MRI examinations with gadobutrol & 75.7 & 84.0 & 23.7 & 26.5 \\
\hline \multicolumn{5}{|l|}{ Malignant lesions $(\mathrm{n}=39)$} \\
\hline MRI examinations with gadoteric acid & 82.4 & 87.0 & 16.2 & 23.0 \\
\hline MRI examinations with gadobutrol & 83.9 & 89.0 & 15.2 & 17.0 \\
\hline MRI examinations with gadobutrol & 42.7 & 41.0 & 24.9 & 48.8 \\
\hline
\end{tabular}

CAD indicates computer-assisted diagnosis; MRI, magnetic resonance imaging; NS, nonsignificant.

worldwide, such as time resolution, $2 \mathrm{D}$ versus $3 \mathrm{D}$ dynamic scanning, and use of parallel imaging or fat suppression. ${ }^{38,39}$ Analyzing 3 different MR imaging protocols, Jansen et $\mathrm{al}^{38}$ found significantly different dynamic initial and postinitial characteristics of breast lesions. Thus, the characterization of breast lesions and description of possible BE can vary due to technical MRI parameters (eg, 2D versus $3 \mathrm{D}$, duration of dynamic $\mathrm{T} 1$-weighted sequences), the injection bolus (eg, amount of saline flush, flow rate, pause after injection), and patient conditions (eg, application of hormone therapy, cardiac insufficiency). Additionally, the reported differences for contrast media in published intraindividual comparisons ${ }^{21-24}$ and the findings of our intraindividual comparison study, with identical technical parameters and patient conditions of both MRI examinations (with gadoteric acid and gadobutrol), revealed that the type of contrast medium can also influence the characterization of breast lesions. Using the same type and dose of contrast agents is therefore particularly important in follow-up MRI examinations, such as monitoring the efficacy of neoadjuvant chemotherapy.

There are certain limitations of our investigation. The number of patients and particularly the included benign lesions are of small

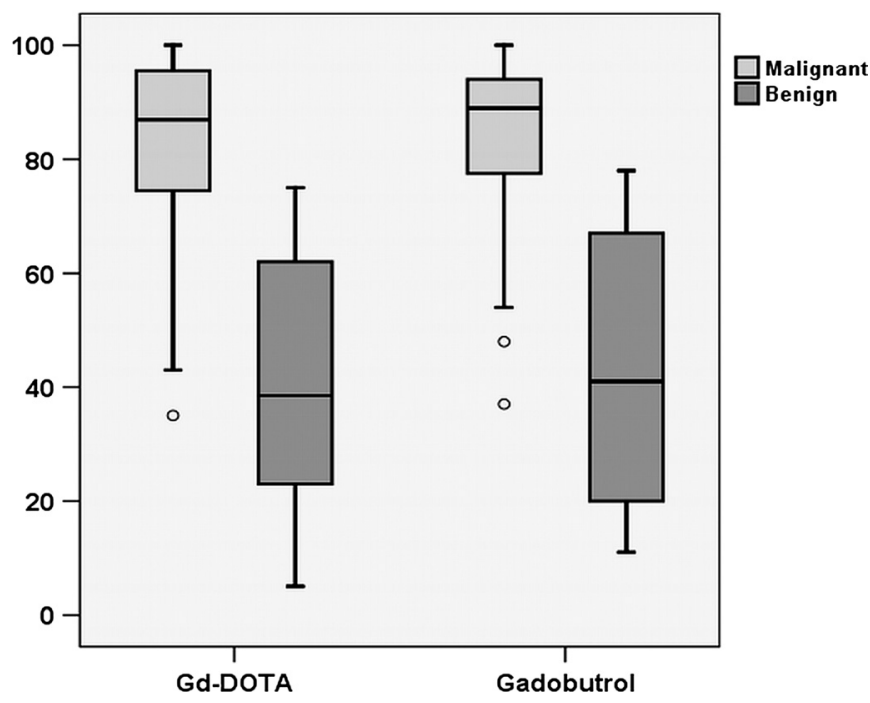

FIGURE 4. Box plots present the calculation of the probability of malignancy (by the MDI) for malignant $(n=39)$ and benign $(n=10)$ lesions by the CAD system. The lesion classifications by the MDI were performed for the MRI examinations with gadoteric acid versus gadobutrol. quantity, as the reference standard was histopathologic verification of masses; lesions that presented as obviously benign on MRI were not histopathologically proven after the MRI examinations and could therefore not be included. The included patient collective is highly selected and small; the findings of this study have to be validated using larger patient cohorts in the future. Another important limitation is the exclusion of non-mass lesions because of configurations of the used CAD system, which results in selection bias and requires further investigations with inclusion of non-mass lesions. To minimize technical effects on dynamic and morphologic MRI parameters, for example, due to multicenter differences in sequence parameters, we decided to implement unique MRI protocol in a single center with consistent clinical, radiological, and histopathologic data. However, because of varying breast sizes, the FOV of the MRI examinations differed between patients, which might influence the morphologic characterization of lesions, although the FOV remained constant within the 2 MRI examinations (with gadoteric acid and gadobutrol) of the same patient. The size of the included masses was quite large, with mean diameter of approximately $25 \mathrm{~mm}$; however, this lesion size is in the range of published studies, evaluating breast MR images by CAD systems. ${ }^{15}$ There exists a lack of data for small lesions, as the correct detection and characterization of small breast lesions remain a specific challenge for CAD systems on breast MRI. ${ }^{52}$

Further limitations of this investigation are that premenopausal and postmenopausal women were included; patients could not discontinue possible hormonal medication for a longer time interval, and premenopausal women did not undergo breast DCE-MRI at the exactly same time points of their menstrual cycle. In a recently published trial, premenopausal women presented higher BE than postmenopausal women did. ${ }^{53}$ However, these study conditions are prevalent in typical clinical settings. The time interval between the 2 MRI examinations was 7 days or less, mostly 1 or 2 days; possible effects of different menstrual cycle phases or other hormonal effects cannot be fully excluded. As BE did not differ between the 2 MRI examinations, hormonal effects might be similar, and thus, the MRI examinations with gadoteric acid versus gadobutrol could be reliably compared.

\section{CONCLUSIONS}

The findings of this prospective investigation indicate that the macrocyclic contrast media gadoteric acid and gadobutrol can be accurately used for breast MRI. Some morphologic and dynamic characterization of histologically verified breast lesions by using gadoteric acid versus gadobutrol differed in an intraindividual, blinded comparison, assessed by human observers and an automatic CAD system. However, the final classification of the breast lesions and the diagnostic accuracy of the MRI examinations were similar between 
gadoteric acid and gadobutrol. Besides the standardization of technical MRI and injection bolus parameters as well as imaging interpretation, the standardization of the applied contrast medium seems to be important for reproducible characterization of breast lesions, particularly with regard to follow-up MRI examinations, such as monitoring the efficacy of neoadjuvant chemotherapy.

\section{ACKNOWLEDGMENT}

The authors thank Toni Vomweg, MD, Heiner Faber, and Dirk Iwamaru, PhD (all Bingen, Germany) for their technical support. The authors thank Marc Dewey, MD, PhD (Berlin, Germany), for his thorough editing of this manuscript. In addition, the authors thank Ulrich Speck, PhD, for scientific advice and Bettina Herwig (both Berlin, Germany) for editorial assistance.

\section{REFERENCES}

1. Ferlay J, Shin HR, Bray F, et al. Estimates of worldwide burden of cancer in 2008: GLOBOCAN 2008. Int J Cancer. 2010;127:2893-2917.

2. Kaiser WA, Zeitler E. MR imaging of the breast: fast imaging sequences with and without Gd-DTPA: preliminary observations. Radiology. 1989;170: 681-686.

3. Orel SG, Schnall MD. MR imaging of the breast for the detection, diagnosis, and staging of breast cancer. Radiology. 2001;220:13-30.

4. Kuhl CK. Current status of breast MR imaging, part 2: clinical applications. Radiology. 2007;244:672-691.

5. Sardanelli F, Podo F, Santoro F, et al. Multicenter surveillance of women at high genetic breast cancer risk using mammography, ultrasonography, and contrastenhanced magnetic resonance imaging (The High Breast Cancer Risk Italian 1 Study): final results. Invest Radiol. 2011;46:94-105.

6. Lehman CD, Gatsonis C, Kuhl CK, et al. MRI evaluation of the contralateral breast in women with recently diagnosed breast cancer. $N$ Engl J Med. 2007; 356:1295-1303

7. Renz DM, Böttcher J, Baltzer PAT, et al. The contralateral synchronous breast carcinoma: a comparison of histology, localization, and magnetic resonance imaging characteristics with the primary index cancer. Breast Cancer Res Treat. 2010;120:449-459.

8. Pediconi F, Miglio E, Telesca M, et al. Effect of preoperative breast magnetic resonance imaging on surgical decision making and cancer recurrence rates. Invest Radiol. 2012;47:128-135.

9. Kuhl CK, Mielcareck P, Klaschik S, et al. Dynamic breast MR imaging: are signal intensity time course data useful for differential diagnosis of enhancing lesions? Radiology. 1999;211:101-110.

10. Renz DM, Diekmann F, Schmitzberger FF, et al. Pharmacokinetic approach for dynamic breast MRI to indicate signal intensity time curves of benign and malignant lesions by using the tumor flow residence time. Invest Radiol. 2013; 48:69-78.

11. Ikeda DM, Hylton NM, Kuhl CK, et al. MRI Breast Imaging Reporting and Data System (BI-RADS) Atlas. 1st ed. Reston, VA: American College of Radiology; 2003.

12. Mahoney MC, Gatsonis C, Hanna L, et al. Positive predictive value of BIRADS MR imaging. Radiology. 2012;264:51-58.

13. Sohns C, Scherrer M, Staab W, et al. Value of the BI-RADS classification in MR-mammography for diagnosis of benign and malignant breast tumors. Eur Radiol. 2011;21:2475-2483.

14. Ikeda DM, Hylton NM, Kinkel K, et al. Development, standardization, and testing of a lexicon for reporting contrast-enhanced breast magnetic resonance imaging studies. J Magn Reson Imaging. 2001;13:889-895.

15. Dorrius MD, Jansen-van der Weide MC, van Ooijen PM, et al. Computer-aided detection in breast MRI: a systematic review and meta-analysis. Eur Radiol. 2011;21:1600-1608.

16. Meinel LA, Stolpen AH, Berbaum KS, et al. Breast MRI lesion classification: improved performance of human readers with a backpropagation neural network computer-aided diagnosis (CAD) system. J Magn Reson Imaging. 2007; 25:89-95.

17. Vomweg TW, Buscema M, Kauczor HU, et al. Improved artificial neural networks in prediction of malignancy of lesions in contrast-enhanced MRmammography. Med Phys. 2003;30:2350-2359.

18. Renz DM, Böttcher J, Diekmann F, et al. Detection and classification of contrast-enhancing masses by a fully automatic computer-assisted diagnosis system for breast MRI. J Magn Reson Imaging. 2012;35:1077-1088.

19. Newell D, Nie K, Chen JH, et al. Selection of diagnostic features on breast MRI to differentiate between malignant and benign lesions using computer-aided diagnosis: differences in lesions presenting as mass and non-mass-like enhancement. Eur Radiol. 2010;20:771-781.

20. Szabó BK, Wiberg MK, Boné B, et al. Application of artificial neural networks to the analysis of dynamic MR imaging features of the breast. Eur Radiol. 2004; $14: 1217-1225$.
21. Martincich L, Faivre-Pierret M, Zechmann CM, et al. Multicenter, double-blind, randomized, intraindividual crossover comparison of gadobenate dimeglumine and gadopentetate dimeglumine for breast MR imaging (DETECT trial). Radiology. 2011;258:396-408.

22. Pediconi F, Catalano C, Occhiato R, et al. Breast lesion detection and characterization at contrast-enhanced MR mammography: gadobenate dimeglumine versus gadopentetate dimeglumine. Radiology. 2005;237:45-56.

23. Pediconi F, Catalano C, Padula S, et al. Contrast-enhanced MR mammography: improved lesion detection and differentiation with gadobenate dimeglumine. AJR Am J Roentgenol. 2008;191:1339-1346.

24. Pediconi F, Kubik-Huch R, Chilla B, et al. Intra-individual randomised comparison of gadobutrol $1.0 \mathrm{M}$ versus gadobenate dimeglumine $0.5 \mathrm{M}$ in patients scheduled for preoperative breast MRI. Eur Radiol. 2013;23:84-92.

25. Bellin MF, Van Der Molen AJ. Extracellular gadolinium-based contrast media: an overview. Eur J Radiol. 2008;66:160-167.

26. Frenzel T, Lengsfeld P, Schirmer H, et al. Stability of gadolinium-based magnetic resonance imaging contrast agents in human serum at $37^{\circ} \mathrm{C}$. Invest Radiol. 2008;43:817-828.

27. Sieber MA, Lengsfeld P, Frenzel T, et al. Preclinical investigation to compare different gadolinium-based contrast agents regarding their propensity to release gadolinium in vivo and to trigger nephrogenic systemic fibrosis-like lesions. Eur Radiol. 2008;18:2164-2173.

28. Pietsch H, Raschke M, Ellinger-Ziegelbauer H, et al. The role of residual gadolinium in the induction of nephrogenic systemic fibrosis-like skin lesions in rats. Invest Radiol. 2011;46:48-56.

29. Broome DR. Nephrogenic systemic fibrosis associated with gadolinium based contrast agents: a summary of the medical literature reporting. Eur J Radiol. 2008;66:230-234

30. Prince MR, Zhang H, Morris M, et al. Incidence of nephrogenic systemic fibrosis at two large medical centers. Radiology. 2008;248:807-816.

31. Rohrer M, Bauer H, Mintorovitch J, et al. Comparison of magnetic properties of MRI contrast media solutions at different magnetic field strengths. Invest Radiol. 2005;40:715-724.

32. Tombach B, Heindel W. Value of 1.0-M gadolinium chelates: review of preclinical and clinical data on gadobutrol. Eur Radiol. 2002;12:1550-1556.

33. Maurer $\mathrm{M}$, Heine $\mathrm{O}$, Wolf $\mathrm{M}$, et al. Tolerability and diagnostic value of gadoteric acid in the general population and in patients with risk factors: results in more than 84,000 patients. Eur J Radiol. 2012;81:885-890.

34. Baltzer PA, Dietzel M, Vag T, et al. Clinical MR mammography: impact of hormonal status on background enhancement and diagnostic accuracy. Rofo. 2011; 183:441-447.

35. Berg WA, Gutierrez L, NessAiver MS, et al. Diagnostic accuracy of mammography, clinical examination, US, and MR imaging in preoperative assessment of breast cancer. Radiology. 2004;233:830-849.

36. Baltzer PA, Dietzel M. Breast lesions: diagnosis by using proton MR spectroscopy at 1.5 and $3.0 \mathrm{~T}$-systematic review and meta-analysis. Radiology. 2013;267: $735-746$.

37. Eyal E, Shapiro-Feinberg M, Furman-Haran E, et al. Parametric diffusion tensor imaging of the breast. Invest Radiol. 2012;47:284-291.

38. Jansen SA, Shimauchi A, Zak L, et al. Kinetic curves of malignant lesions are not consistent across MRI systems: need for improved standardization of breast dynamic contrast-enhanced MRI acquisition. AJR Am J Roentgenol. 2009;193: 832-839.

39. Kuhl C. The current status of breast MR imaging, part I: choice of technique, image interpretation, diagnostic accuracy, and transfer to clinical practice. Radiology. 2007;244:356-378.

40. Kuhl CK, Jost P, Morakkabati N, et al. Contrast-enhanced MR imaging of the breast at 3.0 and $1.5 \mathrm{~T}$ in the same patients: initial experience. Radiology. 2006; 239:666-676.

41. Sardanelli F, Fausto A, Esseridou A, et al. Gadobenate dimeglumine as a contrast agent for dynamic breast magnetic resonance imaging: effect of higher initial enhancement thresholds on diagnostic performance. Invest Radiol. 2008; 43:236-242.

42. Carbonaro LA, Verardi N, Di Leo G, et al. Handling a high relaxivity contrast material for dynamic breast MR imaging using higher thresholds for the initial enhancement. Invest Radiol. 2010;45:114-120.

43. Hadizadeh DR, Von Falkenhausen M, Kukuk GM, et al. Contrast material for abdominal dynamic contrast-enhanced 3D MR angiography with parallel imaging: intraindividual equimolar comparison of a macrocyclic $1.0 \mathrm{M}$ gadolinium chelate and a linear ionic $0.5 \mathrm{M}$ gadolinium chelate. AJR Am J Roentgenol. 2010;194:821-829

44. Kul S, Cansu A, Alhan E, et al. Contrast-enhanced MR angiography of the breast: evaluation of ipsilateral increased vascularity and adjacent vessel sign in the characterization of breast lesions. AJR Am J Roentgenol. 2010;195: 1250-1254.

45. Knopp MV, Weiss E, Sinn HP, et al. Pathophysiologic basis of contrast enhancement in breast tumors. J Magn Reson Imaging. 1999;10: 260-266.

46. Anzalone N, Scarabino T, Venturi C, et al. Cerebral neoplastic enhancing lesions: multicenter, randomized, crossover intraindividual comparison between 
gadobutrol $(1.0 \mathrm{M})$ and gadoterate meglumine $(0.5 \mathrm{M})$ at $0.1 \mathrm{mmol} \mathrm{Gd} / \mathrm{kg}$ body weight in a clinical setting. Eur J Radiol. 2013;82:139-145.

47. Attenberger UI, Runge VM, Morelli JN, et al. Evaluation of gadobutrol, a macrocyclic, nonionic gadolinium chelate in a brain glioma model: comparison with gadoterate meglumine and gadopentetate dimeglumine at $1.5 \mathrm{~T}$, combined with an assessment of field strength dependence, specifically 1.5 versus 3 T. J Magn Reson Imaging. 2010;31:549-555.

48. Vogler H, Platzek J, Schuhmann-Giampieri G, et al. Pre-clinical evaluation of gadobutrol: a new, neutral, extracellular contrast agent for magnetic resonance imaging. Eur J Radiol. 1995;21:1-10.

49. Wegrowski Y, Maquart FX. Involvement of stromal proteoglycans in tumour progression. Crit Rev Oncol Hematol. 2004;49:259-268.
50. Bashir A, Gray ML, Boutin RD, et al. Glycosaminoglycan in articular cartilage: in vivo assessment with delayed $\mathrm{Gd}(\mathrm{DTPA})^{(2-)}$-enhanced MR imaging. Radiology. 1997;205:551-558.

51. Wiener E, Woertler K, Weirich G, et al. Contrast enhanced cartilage imaging: comparison of ionic and non-ionic contrast agents. Eur J Radiol. 2007;63:110-119.

52. Meyer-Baese A, Schlossbauer T, Lange O, et al. Small lesions evaluation based on unsupervised cluster analysis of signal-intensity time courses in dynamic breast MRI. Int J Biomed Imaging. 2009;2009:326924.

53. Scaranelo AM, Carrillo MC, Fleming R, et al. Pilot study of quantitative analysis of background enhancement on breast MR images: association with menstrual cycle and mammographic breast density. Radiology. 2013;267: $692-700$. 